\title{
Analog gravity from field theory normal modes?
}

\author{
Carlos Barceló \\ Physics Department, Washington University, Saint Louis, MO 63130-4899, USA \\ Stefano Liberati \\ Physics Department, University of Maryland, College Park, MD 20742-4111, USA \\ Matt Visserf \\ Physics Department, Washington University, Saint Louis MO 63130-4899, USA
}

(Dated: 2 April 2001; LTEX-ed October 27, 2018)

\begin{abstract}
We demonstrate that the emergence of a curved spacetime "effective Lorentzian geometry" is a common and generic result of linearizing a classical scalar field theory around some non-trivial background configuration. This investigation is motivated by considering the large number of "analog models" of general relativity that have recently been developed based on condensed matter physics, and asking whether there is something more fundamental going on. Indeed, linearization of a classical field theory (that is, a field theoretic "normal mode analysis") results in fluctuations whose propagation is governed by a Lorentzian-signature curved spacetime "effective metric". In the simple situation considered in this paper, (a single classical scalar field), this procedure results in a unique effective metric, which is quite sufficient for simulating kinematic aspects of general relativity (up to and including Hawking radiation). Upon quantizing the linearized fluctuations around this background geometry, the one-loop effective action is guaranteed to contain a term proportional to the Einstein-Hilbert action of general relativity, suggesting that while classical physics is responsible for generating an "effective geometry", quantum physics can be argued to induce an "effective dynamics". The situation is strongly reminiscent of, though not identical to, Sakharov's "induced gravity" scenario, and suggests that Einstein gravity is an emergent low-energy long-distance phenomenon that is insensitive to the details of the high-energy short-distance physics. (We mean this in the same sense that hydrodynamics is a long-distance emergent phenomenon, many of whose predictions are insensitive to the short-distance cutoff implicit in molecular dynamics.)
\end{abstract}

PACS numbers: 04.40.-b; 04.60.-m; 11.10.-z; 45.20.-d; gr-qc/0104001

Keywords: Analog gravity, field theory, normal modes, emergent phenomena

\section{INTRODUCTION}

The idea of building analog models of, and possibly for, general relativity is currently attracting considerable attention [1]. Because of the extreme difficulty (and inadvisability) of working with intense gravitational fields in a laboratory setting, interest has now turned to investigating the possibility of simulating aspects of general relativity - though it is not a priori expected that all features of Einstein gravity can successfully be carried over to the condensed matter realm. Numerous rather different physical systems have now been seen to be useful for developing analog models of general relativity:

1. Dielectric media: A refractive index can be reinterpreted as an effective metric, the Gordon metric. (Gordon [2], Skrotskii [3], Balazs [4], Plebanski [5], de Felice [6], and many others.)

2. Acoustics in flowing fluids: Acoustic black holes, aka "dumb holes". (Unruh [7], Jacobson [8], Visser [9], Liberati et al [10], and many others.)

3. Phase perturbations in Bose-Einstein condensates: Formally similar to acoustic perturbations, and analyzed using the nonlinear Schrodinger equation (Gross-Pitaevskii equation) and Landau-Ginzburg Lagrangian; typical sound speeds are centimetres per second to millimetres per second. (Garay et al [11], Barceló [12] et al.)

\footnotetext{
*arlos@hbar.wustl.edu: http://www.physics.wustl.edu/ carlos; Supported by the Spanish MEC † liberati@physics.umd.edu; http://www2.physics.umd.edu/ liberati; Supported by the US NSF 挖isser@kiwi.wustl.edu; http://www.physics.wustl.edu/ visser; Supported by the US DOE
} 
4. High-refractive-index dielectric fluids ("slow light"): In dielectric fluids with an extremely high group refractive index it is experimentally possible to slow lightspeed to centimetres per second or less. (Leonhardt-Piwnicki [13], Hau et al 14], Visser [15], and others.)

5. Quasi-particle excitations: Fermionic or bosonic quasi-particles in a heterogeneous superfluid environment. (Volovik [16], Kopnin-Volovik [17], Jacobson-Volovik [18], and Fischer [19].)

6. Nonlinear electrodynamics: If the permittivity and permeability themselves depend on the background electromagnetic field, photon propagation can often be recast in terms of an effective metric. (Plebanski [20, Dittrich-Gies [21], Novello et al [22].)

7. Linear electrodynamics: If you do not take the spacetime metric itself as being primitive, but instead view the linear constitutive relationships of electromagnetism as the fundamental objects, one can nevertheless reconstruct the metric from first principles. (Hehl, Obukhov, and Rubilar [23, 24, 25].)

8. Scharnhorst effect: Anomalous photon propagation in the Casimir vacuum can be interpreted in terms of an effective metric. (Scharnhorst [26], Barton [27], Liberati et al 28], and many others.)

9. Thermal vacuum: Anomalous photon propagation in QED at nonzero temperature can be interpreted in terms of an effective metric. (Gies [29].)

10. "Solid state" black holes. (Reznik [30], Corley and Jacobson [31], and others.)

11. Astrophysical fluid flows: Bondi-Hoyle accretion and the Parker wind [coronal outflow] both provide physical examples where an effective acoustic metric is useful, and where there is good observational evidence that acoustic horizons form in nature. (Bondi [32], Parker [33], Moncrief [34], Matarrese [35], and many others.)

12. Other condensed-matter approaches that don't quite fit into the above classification [36, 37.

A literature search as of April 2001 finds well over a hundred scientific articles devoted to one or another aspect of analog gravity and effective metric techniques. The sheer number of different physical situations lending themselves to an "effective metric" description strongly suggests that there is something deep and fundamental going on. Typically these are models of general relativity, in the sense that they provide an effective metric and so generate the basic kinematical background in which general relativity resides; in the absence of any dynamics for that effective metric we cannot really speak about these systems as models for general relativity. However, as we will discuss more fully bellow, quantum effects in these analog models might provide of some sort of dynamics resembling general relativity.

On a related front, (and we'll see the connection soon enough), the particle physics and relativity communities have also seen Lorentz symmetry emerge as a low-energy approximate symmetry in several physical situations:

1. As an infra-red fixed point of the renormalization group in certain non-Lorentz invariant quantum field theories; (Nielsen et al [38]).

2. As a low-momentum approximation to acoustic propagation in the presence of viscosity; (Visser [9]).

3. As a low-momentum approximation to quasi-particle propagation governed by the Bogolubov dispersion relation; (Barceló et al [12]).

4. In certain other quasi-particle dispersion relations (Volovik 39]).

On a third front, the last few years have seen an increasing number of indications that Einstein gravity (and even quantum field theory) may not be as "fundamental" as was once supposed:

1. Induced gravity: In "induced-gravity" models a la Sakharov [40] the dynamics of gravity is an emergent lowenergy phenomenon that is not fundamental physics. In those models the dynamics of gravity (the approximate Einstein equations) is a consequence of the quantum fluctuations of the other fields in the theory. (In induced gravity models gravitation is not fundamental in exactly the same sense that phonons are not fundamental: phonons are collective excitations of condensed matter systems. Phonons are not fundamental particles in the sense of, say, photons. But this should not stop you from quantizing the phonon field as long as you realise you should not take phonons seriously at arbitrarily high momenta.)

2. Effective field theory for gravity: Donoghue [41] has strongly argued that quantum gravity itself should simply be viewed as an effective field theory, in the same sense that the Fermi theory of the weak interactions is an effective field theory - it still makes sense to quantize in terms of gravitons 42, but the high-energy physics is likely to be rather different from what could be guessed based only on observing low-energy excitations, and you should not necessarily take the gravitons seriously at arbitrarily high momenta. 
3. Effective field theory in general: Indeed, even strictly within the confines of particle physics, attitudes towards effective theories seem to be changing - they are now much more likely to be used, at least as computational tools. As long as one has a clear understanding of when to stop taking them seriously effective theories are perfectly good physics even if they are not "fundamental" [43, 44.

These various observations led us to suspect the existence of a general pattern: That the occurrence of something like an approximate Lorentz symmetry, and something like an approximate non-trivial "effective metric" might be an inescapable general consequence of classical and quantum field theories viewed as dynamical systems. In this article we take some important steps in this direction, and indicate the issues that still must be tackled.

Remember that for mechanical systems with a finite number of degrees of freedom small oscillations can always be resolved into normal modes: a finite collection of uncoupled harmonic oscillators. For a classical field theory you would also expect similar behaviour: small deviations from a background solution of the field equations will be resolved into travelling waves; then these travelling waves can be viewed as an infinite collection of harmonic oscillators, or a finite number if the field theory is truncated in the infra-red and ultra-violet, to which you can then apply a normal mode analysis. The physically interesting question is whether this normal mode analysis for field theories can then be reinterpreted in a "geometrically clean" way in terms of some "effective metric" and "effective geometry". We shall show that whenever we are dealing with a single scalar field the answer is definitely yes: Linearization of any Lagrangian-based dynamics, or linearization of any hyperbolic second-order PDE, will automatically lead to an effective Lorentzian geometry that governs the propagation of the fluctuations. [The general situation (multiple scalar fields, or a multi-component vector or tensor) is quite algebraically messy — details of that situation will be deferred for now.]

Once we have developed the notion of a derived "effective metric" based on a linearization procedure, we can certainly consider the effect of quantizing the linearized fluctuations. At one loop the quantum effective action will contain a term proportional to the Einstein-Hilbert action - this is, in modern language, a key portion of Sakharov's "induced gravity" idea [0]. In the closing segment of the present article we argue that the occurrence of not just an "effective metric", but also an "effective geometrodynamics" closely related to Einstein gravity is a largely unavoidable feature of the linearization and quantization process.

\section{LAGRANGIAN ANALYSIS}

Suppose we have a single scalar field $\phi$ whose dynamics is governed by some first-order Lagrangian $\mathcal{L}\left(\partial_{\mu} \phi, \phi\right)$. (By "first-order" we mean that the Lagrangian is some arbitrary function of the field and its first derivatives.) We want to consider linearized fluctuations around some background solution $\phi_{0}(t, \vec{x})$ of the equations of motion, and to this end we write

$$
\phi(t, \vec{x})=\phi_{0}(t, \vec{x})+\epsilon \phi_{1}(t, \vec{x})+\frac{\epsilon^{2}}{2} \phi_{2}(t, \vec{x})+O\left(\epsilon^{3}\right) .
$$

Now use this to expand the Lagrangian around the classical solution $\phi_{0}(t, \vec{x})$ :

$$
\begin{aligned}
\mathcal{L}\left(\partial_{\mu} \phi, \phi\right) & =\mathcal{L}\left(\partial_{\mu} \phi_{0}, \phi_{0}\right)+\epsilon\left[\frac{\partial \mathcal{L}}{\partial\left(\partial_{\mu} \phi\right)} \partial_{\mu} \phi_{1}+\frac{\partial \mathcal{L}}{\partial \phi} \phi_{1}\right]+\frac{\epsilon^{2}}{2}\left[\frac{\partial \mathcal{L}}{\partial\left(\partial_{\mu} \phi\right)} \partial_{\mu} \phi_{2}+\frac{\partial \mathcal{L}}{\partial \phi} \phi_{2}\right] \\
& +\frac{\epsilon^{2}}{2}\left[\frac{\partial^{2} \mathcal{L}}{\partial\left(\partial_{\mu} \phi\right) \partial\left(\partial_{\nu} \phi\right)} \partial_{\mu} \phi_{1} \partial_{\nu} \phi_{1}+2 \frac{\partial^{2} \mathcal{L}}{\partial\left(\partial_{\mu} \phi\right) \partial \phi} \partial_{\mu} \phi_{1} \phi_{1}+\frac{\partial^{2} \mathcal{L}}{\partial \phi \partial \phi} \phi_{1} \phi_{1}\right]+O\left(\epsilon^{3}\right) .
\end{aligned}
$$

It is particularly useful to consider the action

$$
S[\phi]=\int \mathrm{d}^{d+1} x \mathcal{L}\left(\partial_{\mu} \phi, \phi\right),
$$

since doing so allows us to integrate by parts. (Note that the Lagrangian $\mathcal{L}$ is taken to be a tensor density, not a scalar.) We can now use the Euler-Lagrange equations for the background field

$$
\partial_{\mu}\left(\frac{\partial \mathcal{L}}{\partial\left(\partial_{\mu} \phi\right)}\right)-\frac{\partial \mathcal{L}}{\partial \phi}=0
$$

to discard the linear terms (remember we are linearizing around a solution of the equations of motion) and so we get

$$
S[\phi]=S\left[\phi_{0}\right]+\frac{\epsilon^{2}}{2} \int \mathrm{d}^{d+1} x\left[\left\{\frac{\partial^{2} \mathcal{L}}{\partial\left(\partial_{\mu} \phi\right) \partial\left(\partial_{\nu} \phi\right)}\right\} \partial_{\mu} \phi_{1} \partial_{\nu} \phi_{1}+\left(\frac{\partial^{2} \mathcal{L}}{\partial \phi \partial \phi}-\partial_{\mu}\left\{\frac{\partial^{2} \mathcal{L}}{\partial\left(\partial_{\mu} \phi\right) \partial \phi}\right\}\right) \phi_{1} \phi_{1}\right]+O\left(\epsilon^{3}\right) .
$$


Having set things up this way, the equation of motion for the linearized fluctuation is now easily read off as

$$
\partial_{\mu}\left(\left\{\frac{\partial^{2} \mathcal{L}}{\partial\left(\partial_{\mu} \phi\right) \partial\left(\partial_{\nu} \phi\right)}\right\} \partial_{\nu} \phi_{1}\right)-\left(\frac{\partial^{2} \mathcal{L}}{\partial \phi \partial \phi}-\partial_{\mu}\left\{\frac{\partial^{2} \mathcal{L}}{\partial\left(\partial_{\mu} \phi\right) \partial \phi}\right\}\right) \phi_{1}=0 .
$$

This is a second-order differential equation with position-dependent coefficients (these coefficients all being implicit functions of the background field $\left.\phi_{0}\right)$. Following an analysis developed for acoustic geometries (Unruh [7], Visser [9], Liberati et al [10], Barceló et al [12]), which we now apply to this much more general situation, the above can be given a nice clean geometrical interpretation in terms of a d'Alembertian wave equation - provided we define the effective spacetime metric by

$$
\left.\sqrt{-g} g^{\mu \nu} \equiv f^{\mu \nu} \equiv\left\{\frac{\partial^{2} \mathcal{L}}{\partial\left(\partial_{\mu} \phi\right) \partial\left(\partial_{\nu} \phi\right)}\right\}\right|_{\phi_{0}}
$$

Suppressing the $\phi_{0}$ except when necessary for clarity, this implies $[\mathrm{in}(\mathrm{d}+1)$ dimensions, $d$ space dimensions plus 1 time dimension]

$$
(-g)^{(d-1) / 2}=-\operatorname{det}\left\{\frac{\partial^{2} \mathcal{L}}{\partial\left(\partial_{\mu} \phi\right) \partial\left(\partial_{\nu} \phi\right)}\right\}
$$

Therefore

$$
g^{\mu \nu}\left(\phi_{0}\right)=\left.\left(-\operatorname{det}\left\{\frac{\partial^{2} \mathcal{L}}{\partial\left(\partial_{\mu} \phi\right) \partial\left(\partial_{\nu} \phi\right)}\right\}\right)^{-1 /(d-1)}||_{\phi_{0}}\left\{\frac{\partial^{2} \mathcal{L}}{\partial\left(\partial_{\mu} \phi\right) \partial\left(\partial_{\nu} \phi\right)}\right\}\right|_{\phi_{0}}
$$

And, taking the inverse

$$
g_{\mu \nu}\left(\phi_{0}\right)=\left.\left.\left(-\operatorname{det}\left\{\frac{\partial^{2} \mathcal{L}}{\partial\left(\partial_{\mu} \phi\right) \partial\left(\partial_{\nu} \phi\right)}\right\}\right)^{1 /(d-1)}\right|_{\phi_{0}}\left\{\frac{\partial^{2} \mathcal{L}}{\partial\left(\partial_{\mu} \phi\right) \partial\left(\partial_{\nu} \phi\right)}\right\}^{-1}\right|_{\phi_{0}}
$$

We can now write the equation of motion for the linearized fluctuations in the geometrical form

$$
\left[\Delta\left(g\left(\phi_{0}\right)\right)-V\left(\phi_{0}\right)\right] \phi_{1}=0
$$

where $\Delta$ is the d'Alembertian operator associated with the effective metric $g\left(\phi_{0}\right)$, and $V\left(\phi_{0}\right)$ is the background-fielddependent potential

$$
\begin{aligned}
V\left(\phi_{0}\right) & =\frac{1}{\sqrt{-g}}\left(\frac{\partial^{2} \mathcal{L}}{\partial \phi \partial \phi}-\partial_{\mu}\left\{\frac{\partial^{2} \mathcal{L}}{\partial\left(\partial_{\mu} \phi\right) \partial \phi}\right\}\right) \\
& =\left(-\operatorname{det}\left\{\frac{\partial^{2} \mathcal{L}}{\partial\left(\partial_{\mu} \phi\right) \partial\left(\partial_{\nu} \phi\right)}\right\}\right)^{-1 /(d-1)}\left(\frac{\partial^{2} \mathcal{L}}{\partial \phi \partial \phi}-\partial_{\mu}\left\{\frac{\partial^{2} \mathcal{L}}{\partial\left(\partial_{\mu} \phi\right) \partial \phi}\right\}\right) .
\end{aligned}
$$

Thus $V\left(\phi_{0}\right)$ is a true scalar (not a density). Note that the differential equation (11) is automatically formally selfadjoint (with respect to the measure $\sqrt{-g} \mathrm{~d}^{d+1} x$ ).

It is possible to modify the metric by a conformal factor - doing so preserves the causal properties of the Lorentzian geometry but destroys formal self-adjointness. (Nevertheless, one may be willing to pay this price if the payoff is high enough.) Specifically let us define

$$
\tilde{g}_{\mu \nu} \equiv \exp (-2 \theta) g_{\mu \nu}
$$

Then

$$
\left.\sqrt{-\tilde{g}} \tilde{g}^{\mu \nu} \exp [(d-1) \theta] \equiv f^{\mu \nu} \equiv\left\{\frac{\partial^{2} \mathcal{L}}{\partial\left(\partial_{\mu} \phi\right) \partial\left(\partial_{\nu} \phi\right)}\right\}\right|_{\phi_{0}}
$$

A brief computation now yields

$$
\left[\Delta\left(\tilde{g}\left(\phi_{0}\right)\right)+(d-1) \nabla \theta \cdot \nabla-\tilde{V}\left(\phi_{0}\right)\right] \phi_{1}=0
$$


where the inner product is defined in terms of $\tilde{g}$ and now

$$
\tilde{V}\left(\phi_{0}\right) \equiv \exp (2 \theta) V\left(\phi_{0}\right) .
$$

This could be used, for instance, to simplify the potential term at the cost of self-adjointness. Generically, one would wish to preserve formal self adjointness even at the cost of a more complicated potential term, but we emphasize that at the level of causality there is no strong reason for making a fixed choice.

It is important to realise just how general the result is (and where the limitations are): it works for any Lagrangian depending only on a single scalar field and its first derivatives. The linearized PDE will be hyperbolic (and so the linearized equations will have wave-like solutions) if and only if the effective metric $g_{\mu \nu}$ has Lorentzian signature $\pm\left[-,+^{d}\right]$. Observe that if the Lagrangian contains nontrivial second derivatives you should not be too surprised to see terms beyond the d'Alembertian showing up in the linearized equations of motion. Specific examples of this in special cases are already known: for example this happens in the acoustic geometry when you add viscosity (Visser [9]; not really a Lagrangian system but the general idea is the same), or in the quantum geometry of the Bose-Einstein condensate if you keep terms arising from the quantum potential (Barceló et al [12]).

As a specific example of the appearance of effective metrics due to Lagrangian dynamics we mention that inviscid irrotational barotropic hydrodynamics naturally falls into this scheme (which is why, with hindsight, the derivation of the acoustic metric was so relatively straightforward) [7, 9, 12]. In inviscid irrotational barotropic hydrodynamics the lack of viscosity (dissipation) guarantees the existence of a Lagrangian; which a priori could depend on several fields. Since the flow is irrotational $\vec{v}=\nabla \vartheta$ is a function only of the velocity potential, and the Lagrangian is a function only of this potential and the density. Finally the equation of state can be used to eliminate the density leading to a Lagrangian that is a function only of the single field $\vartheta$ and its derivatives.

Note that in all these cases the (fundamental) dimensionality of spacetime is put in by hand — in the present formalism there is no way to determine the fundamental dimensionality dynamically. (Of course in a Kaluza-Klein framework the effective dimensionality can change if some dimensions become small for dynamical reasons.) Also note that $d=1$ space dimensions is special, and the present formulation does not work unless $\operatorname{det}\left(f^{\mu \nu}\right)=1$. This observation can be traced back to the conformal covariance of the Laplacian in $1+1$ dimensions, and implies (perhaps ironically) that the only time the procedure risks failure is when considering a field theory defined on the world sheet of a string-like object.

We next demonstrate that even if you do not have a Lagrangian, it is still possible to extract an "effective metric" for a system with one degree of freedom. (More precisely, we can define a conformal class of effective metrics. We shall see that the analysis is not as geometrically "clean".)

\section{SECOND-ORDER HYPERBOLIC PDE: LINEARIZATION AND GEOMETRICAL INTERPRETATION}

Consider an arbitrary second-order hyperbolic PDE written in the form

$$
F\left(x, \phi, \partial_{\mu} \phi, \partial_{\mu} \partial_{\nu} \phi\right)=0 .
$$

The function $F$ and the field $\phi$ are taken to be real. The PDE does not have to be linear or even quasi-linear. Defining hyperbolicity for such a general equation is not trivial - not even Courant and Hilbert [45] deal with this particular case explicitly. Instead we shall slightly adapt the definitions of Courant and Hilbert, using them to define hyperbolicity for this system in terms of the linearized equation.

So, suppose we linearize around some solution $\phi_{0}$, writing

$$
\phi(t, \vec{x})=\phi_{0}(t, \vec{x})+\epsilon \phi_{1}(t, \vec{x})+O\left(\epsilon^{2}\right) .
$$

Then

$$
\frac{\partial F}{\partial\left(\partial_{\mu} \partial_{\nu} \phi\right)} \partial_{\mu} \partial_{\nu} \phi_{1}+\frac{\partial F}{\partial\left(\partial_{\mu} \phi\right)} \partial_{\mu} \phi_{1}+\frac{\partial F}{\partial \phi} \phi_{1}=0
$$

Thus the fluctuation satisfies a second-order linear PDE with time-dependent and position-dependent coefficients (these coefficients again depend on the background field about which one is linearizing), though the linear PDE looks somewhat different from what we encountered in the Lagrangian analysis. The linearized PDE is said to be hyperbolic if the matrix $\partial F / \partial\left(\partial_{\mu} \partial_{\nu} \phi\right)$ has "Lorentzian" signature $\pm\left[-,{ }^{d}\right]$. This corresponds to standard mathematical usage, as given for instance in volume 2 of Courant and Hilbert [45] or in the Encyclopedic Dictionary of Mathematics [46]. 
To give a geometrical interpretation to the linearized PDE we start by regrouping the coefficients as follows:

$$
\partial_{\mu}\left\{\frac{\partial F}{\partial\left(\partial_{\mu} \partial_{\nu} \phi\right)} \partial_{\nu} \phi_{1}\right\}+\left\{\frac{\partial F}{\partial\left(\partial_{\mu} \phi\right)}-\partial_{\mu}\left(\frac{\partial F}{\partial\left(\partial_{\mu} \partial_{\nu} \phi\right)}\right)\right\} \partial_{\mu} \phi_{1}+\frac{\partial F}{\partial \phi} \phi_{1}=0 .
$$

Now define

$$
f^{\mu \nu} \equiv \frac{\partial F}{\partial\left(\partial_{\mu} \partial_{\nu} \phi\right)}
$$

and

$$
\Gamma^{\mu} \equiv \frac{\partial F}{\partial\left(\partial_{\mu} \phi\right)}-\partial_{\mu}\left(\frac{\partial F}{\partial\left(\partial_{\mu} \partial_{\nu} \phi\right)}\right)
$$

Then

$$
\partial_{\mu}\left\{f^{\mu \nu} \partial_{\nu} \phi_{1}\right\}+\Gamma^{\mu} \partial_{\mu} \phi_{1}+\frac{\partial F}{\partial \phi} \phi_{1}=0
$$

To complete the geometrical interpretation, it is most elegant to modify the formalism developed for Lagrangian dynamics and define a conformal class of metrics by

$$
\Omega^{-(d-1)} \sqrt{-g} g^{\mu \nu}(\phi) \equiv f^{\mu \nu} .
$$

Here $\Omega$ is (for now) a free variable, which will be chosen to simplify the final result. In terms of this metric the PDE becomes

$$
\Omega^{-(d-1)} \sqrt{-g} \Delta \phi_{1}+\left[\Gamma^{\mu}-(d-1) \Omega^{-(d-1)} \sqrt{-g} g^{\mu \nu} \partial_{\nu} \ln \Omega\right] \partial_{\mu} \phi_{1}+\frac{\partial F}{\partial \phi} \phi_{1}=0 .
$$

We now construct the vector

$$
A^{\mu}\left(\phi_{0}\right)=\frac{\Omega^{(d-1)}}{\sqrt{-g}}\left[\Gamma^{\mu}-(d-1) \Omega^{-(d-1)} \sqrt{-g} g^{\mu \nu} \partial_{\nu} \ln \Omega\right]=\frac{\Omega^{(d-1)}}{\sqrt{-g}}\left[\Gamma^{\mu}-(d-1) f^{\mu \nu} \partial_{\nu} \ln \Omega\right]
$$

and define the potential

$$
V\left(\phi_{0}\right)=-\frac{\Omega^{(d-1)}}{\sqrt{-g}} \frac{\partial F}{\partial \phi}
$$

After all this the linearized PDE becomes:

$$
\left[\Delta\left(g\left(\phi_{0}\right)\right)+A^{\mu}\left(\phi_{0}\right) \partial_{\mu}-V\left(\phi_{0}\right)\right] \phi_{1}=0
$$

This is the geometrical formulation we are searching for.

The conformal factor $\Omega$, buried in the definitions of $g_{\mu \nu}, A^{\mu}$, and $V$, is at this stage arbitrary and may be chosen to simplify the formulæ below according to some convenient prescription. In particular, and in contrast to the Lagrangian-based analysis, the linearized PDE is not automatically self-adjoint. Formal self-adjointness is equivalent to being able to choose $A_{\mu}\left(\phi_{0}\right)=0$. This can be done if and only if the covariant vector

$$
B_{\mu}=\left[f^{-1}\right]_{\mu \nu} \Gamma^{\nu}=\left[\left(\frac{\partial F}{\partial\left(\partial_{\bullet} \partial_{\bullet} \phi\right)}\right)^{-1}\right]_{\mu \nu}\left\{\frac{\partial F}{\partial\left(\partial_{\nu} \phi\right)}-\partial_{\sigma}\left(\frac{\partial F}{\partial\left(\partial_{\sigma} \partial_{\nu} \phi\right)}\right)\right\}
$$

is exact. Observe that this vector is defined directly in terms of the coefficients in the linearized PDE, without having to pre-choose the conformal factor $\Omega(\phi)$. If $B_{\mu}$ is exact $\left(B_{\mu}=\partial_{\mu} \Theta\right)$ then we can choose $\Omega$ according to the prescription

$$
\Omega^{d-1}=\exp (\Theta)
$$

This has the effect of fixing the conformal factor $\Omega$ and the metric $g$ in such a way that $A^{\mu}=0$. In this case the linearized PDE is self-adjoint and extremely compact

$$
\left[\Delta\left(g\left(\phi_{0}\right)\right)-V\left(\phi_{0}\right)\right] \phi_{1}=0
$$


where $\Delta$ is the d'Alembertian operator associated with the effective metric $g$, and $V$ is the scalar potential. This finally is identical in form to the equation derived on the basis of the Lagrangian analysis.

While several of the technical details are different from the Lagrangian-based analysis, the basic flavor is the same: The key point is that hyperbolicity of the linearized PDE is defined in terms of the presence of a matrix of indefinite signature $\pm\left[-,+^{d}\right]$. This matrix is enough to define a conformal class of Lorentzian metrics, and picking the "right" member of the conformal class is largely a matter of taste - do whatever makes the "geometrized" equation look cleanest. (In particular if $B_{\mu}$ is exact there is a unique conformal class that makes the linearized PDE self-adjoint.)

It is very important to stress that the hyperbolic character of these systems (versus elliptic, parabolic, or "other") is encoded in the coefficients of the second-derivative terms in (20) [or (11)]. If we assume a hyperbolic equation, then the null cones defined by the metric $g_{\mu \nu}(x)$ contain all the pointwise information about the propagation of waves at arbitrarily large momentum. Equivalently, the null cones determine the propagation of sharp pulses. In fact since the characteristic surfaces, defined by

$$
f(t, \vec{x})=0, \quad \text { with } \quad g^{\mu \nu} \partial_{\mu} f \partial_{\nu} f=0,
$$

are independent of an overall conformal factor in $g^{\mu \nu} \propto \partial F / \partial\left(\partial_{\mu} \partial_{\nu} \phi(q)\right)$ they take into account only the causal structure of the geometry. Wave propagation at low momenta (as opposed to eikonal ray propagation at high momenta) will depend in addition on the conformal factor. That is, additional features of wave propagation will show up when looking at low momenta — where the linear and zero-order terms in the PDE will be crucial.

\section{INDUCED GRAVITY}

At this stage we have derived the existence of a classical background metric $g_{\mu \nu}\left(\phi_{0}\right)$ and linearized fluctuations governed by the equation

$$
\left[\Delta\left(g\left(\phi_{0}\right)\right)-V\left(\phi_{0}\right)\right] \phi_{1}=0
$$

To quantize these linearized fluctuations, and see the manner in which they can back-react on the geometry, we adopt the standard one loop background field formalism. That is, we go all the way back to the classical action given in equation (3) and write the fundamental field $\phi$ as the sum of a background field (not necessarily satisfying the classical equations of motion) plus a quantum fluctuation

$$
\phi=\phi_{\mathrm{b}}+\phi_{\text {quantum }}
$$

Then it is a standard result that integrating out the quantum fluctuations leads to the one-loop result

$$
\Gamma\left[\phi_{\mathrm{b}}\right]=S\left[\phi_{\mathrm{b}}\right]+\frac{1}{2} \hbar \operatorname{tr} \ln \left[\left.\frac{\delta^{2} S}{\delta \phi \delta \phi}\right|_{\phi_{\mathrm{b}}}\right]+O\left(\hbar^{2}\right) .
$$

But in view of our classical analysis performed in section (II) we know that

$$
\left.\frac{\delta^{2} S}{\delta \phi \delta \phi}\right|_{\phi_{\mathrm{b}}}=\Delta\left(g\left(\phi_{\mathrm{b}}\right)\right)-V\left(\phi_{\mathrm{b}}\right)
$$

So we can write

$$
\Gamma\left[g\left(\phi_{\mathrm{b}}\right), \phi_{\mathrm{b}}\right]=S\left[\phi_{\mathrm{b}}\right]+\frac{1}{2} \hbar \operatorname{tr} \ln \left[\Delta\left(g\left(\phi_{\mathrm{b}}\right)\right)-V\left(\phi_{\mathrm{b}}\right)\right]+O\left(\hbar^{2}\right) .
$$

Here the determinant of the differential operator may be defined in terms of zeta functions or heat kernel expansions 47 , 48, 49, 50. Note that we have chosen the notation to emphasize the fact that the effective action depends on the background field in two ways: explicitly through $\phi_{\mathrm{b}}$, and implicitly through $g\left(\phi_{\mathrm{b}}\right)$. The key point (more or less equivalent to Sakharov's "induced gravity" proposal) is that defining the determinant requires both regularization and renormalization, and that doing so introduces counterterms proportional to the first $d / 2$ Seeley-DeWitt coefficients 49 , 50, 51, 52]. The form of these counterterms is well-known, and in fact for the second-order differential operator $\Delta\left(g\left(\phi_{\mathrm{b}}\right)\right)-V\left(\phi_{\mathrm{b}}\right)$ we have

$$
\begin{aligned}
& a_{0}=1 ; \\
& a_{1}=\frac{1}{6} R(g)-V\left(\phi_{\mathrm{b}}\right) ; \\
& a_{2}=\frac{1}{2}\left(\frac{1}{6} R-V\left(\phi_{\mathrm{b}}\right)\right)^{2}+\frac{1}{6} \Delta V\left(\phi_{\mathrm{b}}\right)-\frac{1}{30} \Delta R-\frac{1}{180} R^{\mu \nu} R_{\mu \nu}+\frac{1}{180} R^{\mu \nu \rho \sigma} R_{\mu \nu \rho \sigma} .
\end{aligned}
$$


The higher-order Seeley-DeWitt coefficients are multinomials in the Riemann tensor, its contractions, and covariant derivatives, and in the potential $V\left(\phi_{\mathrm{b}}\right)$ and its covariant derivatives. The zeroth Seeley-DeWitt coefficient $a_{0}$ induces a cosmological constant, while $a_{1}$ induces an Einstein-Hilbert term. There are additional terms proportional to $a_{2}$ [and $a_{3}$ and higher in more than $(3+1)$ dimensions]. The current experimental constraints on these terms are rather weak 53.

In the original version of Sakharov's idea 40, he introduced an explicit ultraviolet cutoff - this approach is equivalent to writing

$$
\ln \left[\Delta\left(g\left(\phi_{\mathrm{b}}\right)\right)-V\left(\phi_{\mathrm{b}}\right)\right]_{\text {cutoff }}=\int_{\text {cutoff }}^{\infty} \frac{d s}{s} \exp \left(-s\left[\Delta\left(g\left(\phi_{\mathrm{b}}\right)\right)-V\left(\phi_{\mathrm{b}}\right)\right]\right) .
$$

The asymptotic expansion of the heat kernel for small $s$ then reads

$$
\left\langle x\left|\exp \left(-s\left[\Delta\left(g\left(\phi_{\mathrm{b}}\right)\right)-V\left(\phi_{\mathrm{b}}\right)\right]\right)\right| x\right\rangle=\frac{1}{(4 \pi s)^{d / 2}}\left[\sum_{n=0}^{N} a_{n} s^{n}+O\left(s^{N+1}\right)\right]
$$

So that

$$
\left\langle x\left|\ln \left[\Delta\left(g\left(\phi_{\mathrm{b}}\right)\right)-V\left(\phi_{\mathrm{b}}\right)\right]_{\text {cutoff }}\right| x\right\rangle=\frac{1}{(4 \pi)^{d / 2}}\left[\sum_{n=0}^{d / 2} a_{n}(\text { cutoff })^{n-d / 2}+\text { finite }\right] .
$$

As usual, one should interpret (cutoff) ${ }^{0}$ as $\ln$ (cutoff) [54. Again we see that the effective action contains terms proportional to a cosmological constant, the Einstein-Hilbert action, and others. It is most useful to organize the terms in the effective action in a gradient expansion in the effective metric and background field. All in all:

$$
\Gamma\left[g\left(\phi_{\mathrm{b}}\right), \phi_{\mathrm{b}}\right]=S\left[\phi_{\mathrm{b}}\right]+\hbar \int \sqrt{-g} \kappa\left[-2 \Lambda+R\left(g\left(\phi_{\mathrm{b}}\right)\right)\right] \mathrm{d}^{d+1} x+\hbar X\left[g\left(\phi_{\mathrm{b}}\right), \phi_{\mathrm{b}}\right]+O\left(\hbar^{2}\right) .
$$

Here $\kappa$ and $\Lambda$ are constants of dimensions (mass) ${ }^{2}$ [that is, (length $)^{-2}$ ] that emerge from the renormalization procedure. Finally $X\left(\phi_{\mathrm{b}}\right)$ denotes all other finite contributions to the renormalized one-loop effective action (including gradient and curvature-squared contributions coming from $a_{2}$, plus possible background-field dependent modifications of $\kappa$ and $\Lambda$ coming from $a_{1}$ and $a_{2}$ ). Phenomenologically, we will want to eventually relate $\kappa$ to the Newton constant, and $\Lambda$ to the cosmological constant. It is the automatic emergence of the Einstein-Hilbert action as part of the one-loop effective action that is the salient point.

Note that our approach is not identical to Sakharov's idea — in his proposal the metric was put in by fiat, but without any intrinsic dynamics; all the dynamics was generated via one loop quantum effects. (Implicit in Sakharov's approach is the assumption that if there is high energy microphysics leading to the notion of the metric, then it should decouple from the low-energy physics; more on this point below.) In our proposal the very existence of the effective metric itself is an emergent phenomenon. In Sakharov's approach the metric was free to be varied at will, leading precisely to the Einstein equations (plus quantum corrections); in our approach the metric is not a free variable and the equations of motion will be a little trickier.

The quantum equations of motion are defined in the usual way by varying with respect to the background $\phi_{\mathrm{b}}$. It is important to remember that the metric is a function of the background field so that it does not make sense to vary the metric independently — we must always evaluate variations using the chain rule. Thus

$$
\left.\frac{\delta \Gamma\left[g\left(\phi_{\mathrm{b}}\right), \phi_{\mathrm{b}}\right]}{\delta \phi_{\mathrm{b}}(x)} \equiv \frac{\delta \Gamma\left[g\left(\phi_{\mathrm{b}}\right), \phi_{\mathrm{b}}\right]}{\delta \phi_{\mathrm{b}}(x)}\right|_{g_{\mathrm{b}}}+\left.\frac{\delta \Gamma\left[g\left(\phi_{\mathrm{b}}\right), \phi_{\mathrm{b}}\right]}{\delta g_{\mu \nu}}\right|_{\phi_{\mathrm{b}}} \frac{\delta g_{\mu \nu}\left(\phi_{\mathrm{b}}\right)}{\delta \phi_{\mathrm{b}}(x)}
$$

Applied to the one-loop action the equations of motion are

$$
\begin{aligned}
\frac{\delta \Gamma\left[g\left(\phi_{\mathrm{b}}\right), \phi_{\mathrm{b}}\right]}{\delta \phi_{\mathrm{b}}(x)}=0 & =\left\{\frac{\delta S\left[\phi_{\mathrm{b}}\right]}{\delta \phi_{\mathrm{b}}}+\left.\hbar \frac{\delta X\left[g\left(\phi_{\mathrm{b}}\right), \phi_{\mathrm{b}}\right]}{\delta \phi_{\mathrm{b}}}\right|_{g_{\mathrm{b}}}\right\} \\
& +\hbar\left\{\kappa\left(\phi_{\mathrm{b}}\right) \sqrt{g}\left[G^{\mu \nu}(g)+\Lambda\left(\phi_{\mathrm{b}}\right) g^{\mu \nu}\right]+\left.\frac{\delta X\left[g\left(\phi_{\mathrm{b}}\right), \phi_{\mathrm{b}}\right]}{\delta g_{\mu \nu}}\right|_{\phi_{0}}\right\} \frac{\delta g_{\mu \nu}\left(\phi_{\mathrm{b}}\right)}{\delta \phi_{\mathrm{b}}(x)}+O\left(\hbar^{2}\right)
\end{aligned}
$$

These are not the Einstein equations, but they are closely related: the first two terms on the RHS are problematic in that they encode the dependence on the original background geometry (typically Minkowski space) implicitly used in 
setting up the fundamental Lagrangian, while the next three terms involve the effective metric and are similar to those obtained in studies of embedded-submanifold versions of general relativity; see for example Regge and Teitelboim [55] and Deser et al [56]. To obtain Einstein-like dynamics we need to suppress the dependence on the original background metric, and write these equations solely in terms of the effective metric $g\left(\phi_{0}\right)$. This can be done if and only if there exists a functional $Z\left[g\left(\phi_{0}\right)\right]$, which depends on $\phi_{0}$ only implicitly via the effective metric, such that

$$
\int \mathcal{L}\left[\phi_{\mathrm{b}}\right] \mathrm{d}^{d+1} x+\hbar X\left[g\left(\phi_{\mathrm{b}}\right), \phi_{\mathrm{b}}\right]=\hbar Z\left[g\left(\phi_{\mathrm{b}}\right)\right]+O\left(\hbar^{2}\right) .
$$

This is, very definitely, a powerful restriction of the theory under consideration; but it appears to be the minimal condition for something similar to Einstein dynamics to arise. In particular we view this particular hurdle as the single biggest issue facing the "induced gravity" proposal, though some particular implementations of this idea may skirt the issue. For instance, consider Volovik's proposals to extract the dynamics of Einstein gravity from condensed matter quasiparticle excitations [39]: Volovik essentially argues that certain theories might exhibit "one-loop dominance" in the sense that the one-loop physics dominates over the zero loop physics. In contrast, Sakharov implicitly assumes that whatever the microphysics is, it has effectively decoupled from the low energy effective metric. Our expression in equation (48) above is an explicit characterization of what this decoupling condition should be. Note that it is quite sufficient for our purposes if this constraint holds as an approximation for some region in field space surrounding the metrics of interest; it does not need to be a global constraint on the theory. Given this constraint the background geometry (the microphysics) decouples from the effective metric (the macrophysics) and we have

$$
\left\{\kappa\left[G^{\mu \nu}(g)+\Lambda g^{\mu \nu}\right]+\frac{1}{\sqrt{g}} \frac{\delta Z\left[g\left(\phi_{\mathrm{b}}\right)\right]}{\delta g_{\mu \nu}}\right\} \frac{\delta g_{\mu \nu}\left(\phi_{\mathrm{b}}\right)}{\delta \phi_{\mathrm{b}}(x)}=O(\hbar) .
$$

The $\delta Z[g] / \delta g$ term now encodes three separate terms form equation (47) and denotes the type of "curvature squared" corrections to the Einstein equations that are commonly encountered in string theory (indeed in almost any candidate theory for quantum gravity) and in the usual implementation of Sakharov's approach. Additionally, it must be emphasised that because of the contraction with the $\delta g_{\mu \nu}\left(\phi_{\mathrm{b}}\right) / \delta \phi_{\mathrm{b}}(x)$ these are not the usual Einstein equations, though they are certainly implied by the (curvature enhanced) Einstein equations. It is in this sense that we can begin to see the structure of Einstein gravity emerging from this field-theoretic normal mode analysis.

\section{DISCUSSION}

In this paper we have provided two key developments:

1. We have shown that the emergence of an "effective metric", in the sense that this notion is used in the so-called "analog models" of general relativity, is a rather generic feature of the linearization process. While the existence of an effective metric by itself does not allow you to simulate all of Einstein gravity, it allows one to do quite enough to be really significant - in particular it seems that the existence of an effective Lorentzian metric is really all that is in principle needed to obtain simulations of the Hawking radiation effect [7, 57, 58. In this regard, the major technical limitation of the current analysis is that it is limited to a single scalar field; extensions of this idea involve both some technical subtleties and some new physics, and we shall discuss that scenario more fully elsewhere. The major piece of additional physics is the possible presence of birefringence, or more generally "multi-refringence", with different normal modes possibly reacting to different metrics. The Eötvös experiment [the observational universality of free fall to extremely high accuracy] indicates that all the physical fields coupling to ordinary bulk matter "see" to high precision the same metric, allowing us to formulate the Einstein Equivalence principle and speak of the metric of spacetime. Thus in extending the notion of effective geometry to a system with many degrees of freedom, experiment tells us that we should seek conditions that would naturally serve to suppress birefringence. Only in that case would it make sense to speak of a unique spacetime metric (or at worst, of multiple almost-degenerate metrics).

2. By invoking one-loop quantum effects, we can argue that something akin to Sakharov's induced gravity scenario is operative: in particular we can generically argue that there is a term in the quantum effective action proportional to the Einstein-Hilbert action. However because of the technical assumption that the effective metric depends on the background only via the single scalar field $\phi_{0}(x)$ we have not been able to reproduce full Einstein gravity, though certainly have some extremely suggestive results along this line. Additional issues that are definitely worth future exploration are the physical import of the fine tuning used to decouple the effective metric from the background [equation (48)], the question of going beyond the linearized approximation [that is, beyond one loop], and whether the addition of extra fields helps one to obtain a better approximation to full Einstein 
gravity - this because you would get one equation of motion per background field, so with six or more fields you would expect to be able to explore the full algebraic structure of the metric. So adding extra fields, which is technically a hindrance in the kinematical part of the program (developing the effective metric formalism), should in compensation allow one to more closely approach the dynamics of Einstein gravity.

In summary: The full generality of the situations under which effective metrics are encountered is truly remarkable, and the extent to which the resulting analog models seem able to reproduce key aspects of Einstein gravity is even more remarkable. The physics of these systems is fascinating, and the potential for laboratory investigation of models close to (but not necessarily identical to) Einstein gravity is extremely encouraging. Our interpretation of these results is that they provide suggestive evidence that what we call Einstein gravity (general relativity) is an almost automatic low-energy consequence of almost any well behaved quantum field theory: the dimensionality of spacetime is put in by hand, but the occurrence of an effective metric is almost automatic (even in the classical theory), while the presence of Einstein-like dynamics can plausibly be engendered by one-loop quantum effects.

\section{Acknowledgements}

The research of Matt Visser was supported by the US Department of Energy. Stefano Liberati was supported by the US National Science Foundation. Carlos Barceló was supported by the Spanish Ministry of Education and Culture (MEC). The authors are indebted to Grigori Volovik for the clear presentation of his ideas on induced gravity in superfluid quasiparticle systems as developed in his lectures at the Workshop on "Analog Models of General Relativity" [1]. The authors are particularly grateful to Sebastiano Sonego for extensive discussions and input; and also wish to thank Ted Jacobson for his interest and comments.

[1] Workshop on "Analog Models of General Relativity" (Rio de Janeiro, October, 2000); http://www.physics.wustl.edu/ visser/Analog

[2] W. Gordon, "Zur Lichtfortpflanzung nach der Relativitätstheorie", Ann. Phys. Leipzig 72, 421 (1923).

[3] G.V. Skrotskii, "The influence of gravitation on the propagation of light", Soviet Physics Doklady 2, 226 (1957).

[4] N.L. Balazs, "Effect of a gravitational field, due to a rotating body, on the plane of polarization of an electromagnetic wave", Phys. Rev. 110, 236 (1958).

[5] J. Plebanski, "Electromagnetic waves in gravitational fields", Phys. Rev. 118, 1396 (1960).

[6] F. de Felice, "On the gravitational field acting as an optical medium", Gen. Rel. Grav. 2347 (1971).

[7] W.G. Unruh, "Experimental black hole evaporation?", Phys. Rev. Lett. 46, 1351 (1981); "Dumb holes and the effects of high frequencies on black hole evaporation", Phys. Rev. D 51, 2827 (1995) [gr-qc/9409008]. (Title changed in journal: "Sonic analog of black holes and...")

[8] T. Jacobson, "Black hole evaporation and ultrashort distances", Phys. Rev. D 44, 1731 (1991); "Black hole radiation in the presence of a short distance cutoff", Phys. Rev. D 48, 728 (1993) hep-th/9303103.

[9] M. Visser, "Acoustic propagation in fluids: An Unexpected example of Lorentzian geometry", gr-qc/9311028; "Acoustic black holes: Horizons, ergospheres, and Hawking radiation", Class. Quantum Grav. 15, 1767 (1998) gr-qc/9712010; "Acoustic black holes", gr-qc/9901047.

[10] S. Liberati, S. Sonego and M. Visser, "Unexpectedly large surface gravities for acoustic horizons?" Class. Quantum Grav. 17, 2903 (2000) gr-qc/0003105.

[11] L. J. Garay, J. R. Anglin, J. I. Cirac and P. Zoller, "Black holes in Bose-Einstein condensates", Phys. Rev. Lett. 85, 4643 (2000) gr-qc/0002015; "Sonic black holes in dilute Bose-Einstein condensates", Phys. Rev. A 63, 023611 (2001) gr-qc/0005131.

[12] C. Barceló, S. Liberati and M. Visser, "Analog gravity from Bose-Einstein condensates", Class. Quantum Grav. 18, 1137 (2001) gr-qc/0011026.

[13] U. Leonhardt and P. Piwnicki, "Optics of nonuniformly moving media", Phys. Rev. A 60, 4301 (1999) physics/9906038; "Relativistic effects of light in moving media with extremely low group velocity", Phys. Rev. Lett. 84, 822 (2000) condmat/9906332; "Reply to the Comment on Relativistic Effects of Light in Moving Media with Extremely Low Group Velocity", Phys. Rev. Lett. 85, 5253 (2000), gr-qc/0003016.

[14] L. V. Hau, S. E. Harris, Z. Dutton, and C. H. Behroozi, "Light speed reduction to 17 metres per second in an ultracold atomic gas", Nature 397, 594 (1999).

[15] M. Visser, "Comment on Relativistic effects of light in moving media with extremely low group velocity", Phys. Rev. Lett. 85, 5252 (2000) gr-qc/0002011.

[16] G. E. Volovik, "Simulation of quantum field theory and gravity in superfluid ${ }^{3}$ He", Low Temp. Phys. (Kharkov) 24, 127 (1998) cond-mat/9706172. "Gravity of monopole and string and gravitational constant in ${ }^{3}$ He-A", Pisma Zh. Eksp. Teor. Fiz. 67, 666 (1998); JETP Lett. 67, 698 (1998) cond-mat/9804078. "Links between gravity and dynamics of quantum liquids", gr-qc/0004049. 
[17] N. B. Kopnin and G. E. Volovik, "Critical velocity and event horizon in pair-correlated systems with "relativistic" fermionic quasiparticles", Pisma Zh. Eksp. Teor. Fiz. 67, 124 (1998) cond-mat/9712187.

[18] T. A. Jacobson and G. E. Volovik, "Effective spacetime and Hawking radiation from moving domain wall in thin film of He-3-A", Pisma Zh. Eksp. Teor. Fiz. 68, 833 (1998) gr-qc/9811014; "Event horizons and ergoregions in He-3", Phys. Rev. D 58, 064021 (1998).

[19] U.R. Fischer, "Motion of Quantized Vortices as Elementary Objects", Ann. Phys. 278, 62 (1999) cond-mat/9907457; U.R. Fischer and G.E. Volovik, "Thermal quasi-equilibrium states across Landau horizons in the effective gravity of superfluids", Int. J. Mod. Phys. D 10, 57 (2001) gr-qc/0003017.

[20] J. Plebanski, Lectures on nonlinear electrodynamics, Nordita, Copenhagen, (1970).

[21] W. Dittrich and H. Gies, "Light propagation in nontrivial QED vacua", Phys. Rev. D 58, 025004 (1998) hep-ph/9804375.

[22] M. Novello, V. A. De Lorenci, J. M. Salim and R. Klippert, "Geometrical aspects of light propagation in nonlinear electrodynamics", Phys. Rev. D 61, 045001 (2000) gr-qc/9911085. V. A. De Lorenci, R. Klippert, M. Novello and J. M. Salim, "Light propagation in non-linear electrodynamics", Phys. Lett. B 482, 134 (2000) gr-qc/0005049].

[23] Y. N. Obukhov and F. W. Hehl, "Spacetime metric from linear electrodynamics", Phys. Lett. B 458, 466 (1999) grqc/9904067.

[24] F. W. Hehl, Y. N. Obukhov and G. F. Rubilar, "Spacetime metric from linear electrodynamics. II", gr-qc/9911096.

[25] Y. N. Obukhov, T. Fukui and G. F. Rubilar, "Wave propagation in linear electrodynamics", Phys. Rev. D 62, 044050 (2000) gr-qc/0005018].

[26] K. Scharnhorst, "On propagation of light in the vacuum between plates", Phys. Lett. B 236, 354-359 (1990). "The velocities of light in modified QED vacua", Ann. Phys. (Leipzig) 7, 700-709 (1998) hep-th/9810221.

[27] G. Barton, "Faster-than-c light between parallel mirrors. The Scharnhorst effect rederived", Phys. Lett. B 237, 559-562 (1990); G. Barton and K. Scharnhorst, "QED between parallel mirrors: light signals faster than $c$, or amplified by the vacuum", J. Phys. A 26, 2037-2046 (1993).

[28] S. Liberati, S. Sonego and M. Visser, "Scharnhorst effect at oblique incidence", Phys. Rev. D 63, 085003 (2001) quant$\mathrm{ph} / 0010055$.

[29] H. Gies, "Light cone condition for a thermalized QED vacuum", Phys. Rev. D 60, 105033 (1999) hep-ph/9906303.

[30] B. Reznik, "Trans-Planckian tail in a theory with a cutoff", Phys. Rev. D 55, 2152 (1997) gr-qc/9606083. "Origin of the thermal radiation in a solid-state analog of a black hole", Phys. Rev. D 62, 0440441 (2000) ggr-qc/9703076.

[31] S. Corlev and T. Jacobson, "Hawking Spectrum and High Frequency Dispersion", Phys. Rev. D 54, 1568 (1996) [hepth/9601073; S. Corley, "Particle creation via high frequency dispersion", Phys. Rev. D 55, 6155 (1997); "Computing the spectrum of black hole radiation in the presence of high frequency dispersion: An analytical approach", Phys. Rev. D 57, 6280 (1998) hep-th/9710075.

[32] H. Bondi, "On spherically symmetric accretion", Mon. Not. Roy. Astron. Soc. 112, 195-204 (1952).

[33] E.N. Parker, "Dynamical properties of stellar coronas and stellar winds V. Stability and wave propagation", Ap. J. 143, 32 (1966).

[34] V. Moncrief, "Stability of a stationary, spherical accretion onto a Schwarzschild black hole", Ap. J. 235, 1038 (1980).

[35] S. Matarrese, "Perturbations of an irrotational perfect fluid", in Proceedings of the 4'th Italian conference on general relativity and the physics of gravitation, (Florence, 10-13 October 1984), pp 283-287; "Phonons in a relativistic perfect fluid", in Proceedings of the 4'th Marcel Grossmann meeting on General Relativity, R. Ruffini (ed.), (Elsevier, 1986), pp 1591-1595; "On the classical and quantum irrotational motions of a relativistic perfect fluid. I. Classical Theory", Proc. R. Soc. Lond. A 40153 (1985).

[36] G. J. Stephens and B. L. Hu, "Notes on black hole phase transitions", gr-qc/0102052.

[37] G. Chapline, E. Hohlfeld, R. B. Laughlin and D. I. Santiago, "Quantum phase transitions and the breakdown of classical general relativity", gr-qc/0012094.

[38] H. B. Nielsen and I. Picek, "Lorentz Noninvariance", Nucl. Phys. B 211, 269 (1983).

S. Chadha and H. B. Nielsen, "Lorentz Invariance As A Low-Energy Phenomenon", Nucl. Phys. B 217, 125(1983).

[39] G. E. Volovik, "Induced gravity in superfluid He-3", J. Low. Temp. Phys. 113, 667 (1997) cond-mat/9806010; "Superfluid analogies of cosmological phenomena", gr-qc/0005091; "Reentrant violation of special relativity in the low-energy corner", hep-ph/0101286.

[40] A. Sakharov, "Vacuum quantum fluctuations in curved space and the theory of gravitation", Soviet Physics Doklady, 12, 1040 (1968).

[41] J. F. Donoghue, "Quantum general relativity is an effective field theory", PRINT-96-198 (MASS.U.,AMHERST) Talk presented at the 10th International Conference on Problems of Quantum Field Theory. Alushta, Ukraine, 13-17 May 1996; "Gravity and Effective Field Theory: A Talk for Phenomenologists", hep-ph/9512287; "Introduction to the Effective Field Theory Description of Gravity", gr-qc/9512024 "The Ideas of gravitational effective field theory", hep-th/9409143; "General relativity as an effective field theory: The leading quantum corrections", Phys. Rev. D 50, 3874 (1994) grqc/9405057.

[42] R. P. Feynman, F. B. Morinigo, W. G. Wagner and B. Hatfield, "Feynman lectures on gravitation", Reading, USA: Addison-Wesley (1995) $232 \mathrm{p}$. (The advanced book program).

[43] S. Weinberg, "What is quantum field theory, and what did we think it was?", hep-th/9702027.

[44] S. Weinberg, The quantum theory of fields, (Cambridge, England, 1999).

[45] R. Courant and D. Hilbert, Methods of Mathematical Physics, Vol II, Wiley, John and Sons, (1990).

[46] Encyclopedic Dictionary of Mathematics, K. Ito, K. Ito and N.S. Ugakkai (Editors), 2nd ed, MIT Press (1987).

[47] J. S. Dowker and R. Critchley, "Effective Lagrangian And Energy Momentum Tensor In De Sitter Space", Phys. Rev. D 
13, $3224(1976)$

[48] S. W. Hawking, "Zeta Function Regularization Of Path Integrals In Curved Space-Time", Commun. Math. Phys. 55, 133 (1977).

[49] S. Blau, M. Visser and A. Wipf, "Zeta Functions And The Casimir Energy", Nucl. Phys. B 310,163 (1988).

[50] N.D. Birrell and P.C.W. Davies, Quantum Fields in curved space, Cambridge University Press, Cambridge (1982).

[51] R. Utiyama and B. S. DeWitt, "Renormalization Of A Classical Gravitational Field Interacting With Quantized Matter Fields", J. Math. Phys. 3, 608 (1962); B. S. DeWitt, Dynamical theory of group and fields, Gordon and Breach, New York (1965); "Quantum field theory in curved spacetime", Phys. Rep. 19 C, 295 (1975).

[52] T. S. Bunch and L. Parker, "Feynman Propagator In Curved Space-Time: A Momentum Space Representation", Phys. Rev. D 20, 2499 (1979).

[53] M. B. Mijic, M. S. Morris and W. Suen, "The $R^{2}$ Cosmology: Inflation Without A Phase Transition", Phys. Rev. D 34, 2934 (1986); see esp p 2944.

[54] C. Itzykson and J.B. Zuber, Quantum field theory, McGraw-Hill, New York (1980).

[55] T. Regge and C. Teitelboim, "General Relativity a la string: a progress report", in Proceedings of the Marcel Grossman meeting, Trieste (1975).

[56] S. Deser, F.A.E. Pirani and D.C. Robinson, "New embedding model of general relativity", Phys. Rev. D 14, 3301 (1976).

[57] S. W. Hawking, "Particle Creation By Black Holes", Commun. Math. Phys. 43, 199 (1975).

[58] M. Visser, "Hawking radiation without black hole entropy", Phys. Rev. Lett. 80, 3436 (1998) gr-qc/9712016]. 